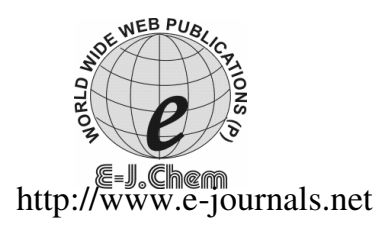

ISSN: 0973-4945; CODEN ECJHAO

E-Journal of Chemistry 2011, 8(2), 809-814

\title{
Synthesis, Characterization and Spectral Studies of Noble Heterobinuclear Complexes of Transition Metal Ions and their Biological Activity
}

\author{
NETRA PAL SINGH* and ABHAY NANDA SRIVASTAVA \\ Department of Chemistry \\ Meerut College Meerut, U.P, India \\ netrapal_chem@yahoo.com
}

Received 17 July 2010; Accepted 5 September 2010

\begin{abstract}
Some noble heterobinuclear complexes of transition metal ions with bis(salicylaldehyde)malonyl-dihydrazone in the presence of 5-nitroindazole $\mathrm{Cu}$ (II) / $\mathrm{Ni}(\mathrm{II})$ - chloride of the type $\left[\mathrm{ML}_{1} \mathrm{ML}_{2} \mathrm{Cl}_{2}\right]$ or $\left[\mathrm{ML}_{1} \mathrm{FeL}_{2} \mathrm{Cl}_{2}\right] \mathrm{Cl}$, where $\mathrm{M}=\mathrm{Ni}(\mathrm{II}), \mathrm{Cu}(\mathrm{II})$ and $\mathrm{M}^{\prime}=\mathrm{Mn}(\mathrm{II}), \mathrm{Co}(\mathrm{II})$, have been prepared. All the complexes have been characterized by IR, UV vis and EPR spectroscopy, elemental analysis, magnetic moment and molar conductance measurement. Spectral studies and magnetic moment measurement in DMF suggest the covalent nature of the complexes, except the $\left[\mathrm{ML}_{1} \mathrm{FeL}_{2} \mathrm{Cl}_{2}\right] \mathrm{Cl}$ complex which is 1:1 electrolyte. An octahedral geometry is proposed for $\mathbf{M}^{`}$ and square planer for $\mathrm{M}$ for the heterobinuclear complexes. The low value of magnetic moment and overlapping EPR signals are due to spin crossover since both of the metals have unpaired electrons with same molecular symmetry. The lowering of the magnetic moment has been discussed. The biological activity (antifungal and antibacterial) of the represented compounds has been studied.
\end{abstract}

Keywords: Heterobinuclear complexes, Malonyldihydrazone, 5-Nitroindazole, Biological activity.

\section{Introduction}

Many of the divalent metal ions are widely presented in vivo as trace elements and essential for the living organism to maintain and regulate biological activities ${ }^{1-2}$. There has been a great interest in the synthesis of heterobinuclear complexes for their interesting magnetic properties $^{3-4}$ and active sites of biomolecules ${ }^{5}$. Heterobinuclear bridged complexes can be formed in stepwise fashion from a mononuclear compound which contains a dangling ligand. The first spin crossover complexes were reported by Tabassum et al. ${ }^{6}$. These complexes are also of interest of bioinorganic chemistry due to the importance of the structurally similar porphyrin complexes with unsymmetrical axial ligation ${ }^{7-9}$. The aim of this work is preparation 
and characterization of heterobinuclear complexes of $\mathrm{Fe}(\mathrm{III}), \mathrm{Co}(\mathrm{II}), \mathrm{Mn}(\mathrm{II}), \mathrm{Cu}(\mathrm{II})$ and $\mathrm{Ni}(\mathrm{II})$. Many other works have been done earlier by various chemists which show current importance and interest of coordination chemistry of transition metal ions ${ }^{10-13}$.

\section{Experimental}

All the chemicals used in this work were analytical grade. Hydrated $\mathrm{Mn}(\mathrm{II}), \mathrm{Co}(\mathrm{II}), \mathrm{Ni}(\mathrm{II})$, $\mathrm{Cu}$ (II) and $\mathrm{Fe}(\mathrm{III})$ chloride (BDH), 5-nitroindazole (Fluka), DMSO, DMF, acetonitrile, malonyl dihydrazone, salicylaldehyde and ethanol. Double distilled water was used throughout the experiment. The transition metal complexes of 5-nitroindazole and bis(salicylaldehyde)malonyl hydrazone were prepared as per the method reported earlier ${ }^{14-15}$.

Preparation of [ $\left.\mathrm{MnL}_{1} \mathrm{NiL}_{2} \mathrm{Cl}_{2}\right]$

A solution of $\mathrm{MnL}_{1}(0.393 \mathrm{~g}, 1 \mathrm{mmol})$ in DMF $(15 \mathrm{~mL})$ was added to the solution of $\mathrm{NiL}_{2} \mathrm{Cl}_{2}(0.455 \mathrm{~g}, 1 \mathrm{mmol})$ and refluxed for $10 \mathrm{~h}$ and then kept in refrigerator overnight. A light pink colour product was formed which was filtered and washed with ethanol, ether and dried in vacuo.

\section{Preparation of $\left[\mathrm{MnL}_{1} \mathrm{CuL}_{2} \mathrm{Cl}_{2}\right]$}

This compound was prepared by using same procedure as above.

\section{Preparation of $\left[\mathrm{CoL}_{1} \mathrm{NiL}_{2} \mathrm{Cl}_{2}\right]$}

A solution of $\mathrm{CoL}_{1}(0.397 \mathrm{~g}, 1 \mathrm{mmol})$ in dry DMF $(15 \mathrm{~mL})$ was refluxed with a methanolic solution $(15 \mathrm{~mL})$ of $\mathrm{NiL}_{2} \mathrm{Cl}_{2}(0.455 \mathrm{~g}, 1 \mathrm{mmol})$. The purple colour solution of $\mathrm{CoL}_{1}$ turned blue on addition of the solution of $\mathrm{NiL}_{2} \mathrm{Cl}_{2}$. A light yellow coloured product was precipitated on refluxing for $8 \mathrm{~h}$. The compound was filtered, washed with ethanol, ether and dried in vacuo.

\section{Preparation of [ $\mathrm{CoL}_{1} \mathrm{CuL}_{2} \mathrm{Cl}_{2}$ ]}

This compound was prepared by using same procedure as above.

Preparation of $\left[\mathrm{NiL}_{1} \mathrm{FeL}_{2} \mathrm{Cl} \mathrm{l}_{2}\right] \mathrm{Cl}$

A solution of $\mathrm{NiL}_{1}(0.397 \mathrm{~g}, 1 \mathrm{mmol})$ in methanol $(15 \mathrm{~mL})$ was treated with a solution of $\left[\mathrm{FeL}_{2} \mathrm{Cl}_{2}\right] \mathrm{Cl}(0.488 \mathrm{~g}, 1 \mathrm{mmol})$ in dry DMF $(15 \mathrm{~mL})$. The resultant solution was refluxed for $20 \mathrm{~h}$. A brown product precipitated. The complex was filtered, washed with ethanol, ether and dried in vacuo.

\section{Preparation of $\left[\mathrm{CuL}_{1} \mathrm{FeL}_{2} \mathrm{Cl} \mathrm{l}_{2}\right] \mathrm{Cl}$}

This complex was prepared by using same procedure as above.

\section{Results and Discussion}

The complexes were prepared according to the following chemical equations-

$$
\begin{aligned}
& \mathrm{MnL}_{1}+\mathrm{NiL}_{2} \mathrm{Cl}_{2} / \mathrm{CuL}_{2} \mathrm{Cl}_{2} \underset{-2 \mathrm{HCl}}{\stackrel{\mathrm{DMF}}{\longrightarrow}} \mathrm{MnL}_{1} \cdot \mathrm{NiLCl}_{2} / \mathrm{CuL}_{2} \mathrm{Cl}_{2} \\
& \mathrm{CoL}_{1}+\mathrm{NiL}_{2} \mathrm{Cl}_{2} / \mathrm{CuL}_{2} \mathrm{Cl}_{2} \underset{-2 \mathrm{HCl}}{\stackrel{\mathrm{DMF}}{\longrightarrow}} \mathrm{CoL}_{1} \cdot \mathrm{NiL}_{2} \mathrm{Cl}_{2} / \mathrm{CuL}_{2} \mathrm{Cl}_{2} \\
& \mathrm{Ni} / \mathrm{Cu}-\mathrm{L}_{1}+\left[\mathrm{FeL}_{2} \mathrm{Cl}_{2}\right] \mathrm{Cl} \underset{-2 \mathrm{HCl}}{\stackrel{\mathrm{DMF}}{\rightarrow}}\left[\mathrm{Ni} / \mathrm{Cu}-\mathrm{L}_{1} \cdot \mathrm{Fe}(\mathrm{III}) \mathrm{L}_{2} \mathrm{Cl}_{2}\right] \mathrm{Cl}
\end{aligned}
$$

Where, $\mathrm{L}_{1}=$ bis(salicylaldehyde)malonyl hydrazone, $\mathrm{L}_{2}=5$-nitroindazole. 
Analytical data are given in Table 1. All the complexes are soluble in DMF and DMSO. Molar conductance values are measured in DMF solvent and show non- electrolyte nature of complexes, except [NiL1FeL2Cl2]Cl and [CuL1FeL2Cl2]Cl which are 1:1 electrolyte. (Figure1.)

Table 1. Analytical data of heterobinuclear complexes

\begin{tabular}{|c|c|c|c|c|c|c|c|}
\hline \multirow{2}{*}{ Complexes } & \multirow{2}{*}{$\begin{array}{l}\text { Molecular Formula } \\
\text { (Formula weight) }\end{array}$} & \multirow{2}{*}{ Colour } & \multirow{2}{*}{$\begin{array}{l}\text { M.P., } \\
{ }^{\circ} \mathrm{C}\end{array}$} & \multirow{2}{*}{$\begin{array}{c}\text { Yield, } \\
\%\end{array}$} & \multicolumn{3}{|c|}{ Calcd. found $\%$} \\
\hline & & & & & $\mathrm{C}$ & $\mathrm{H}$ & $\mathrm{N}$ \\
\hline$[\mathrm{Mn}]$ & $\begin{array}{c}\mathrm{C}_{31} \mathrm{H}_{24} \mathrm{Cl}_{2} \mathrm{MnN}_{10} \mathrm{O}_{8} \mathrm{Ni} \\
(849.15)\end{array}$ & & 326 & 32 & $\begin{array}{c}43.85 \\
(43.88)\end{array}$ & $\begin{array}{c}2.85 \\
(2.88)\end{array}$ & $\begin{array}{l}16.50 \\
(16.54)\end{array}$ \\
\hline$[\mathrm{Mr}$ & $\begin{array}{c}\mathrm{C}_{31} \mathrm{H}_{24} \mathrm{Cl}_{2} \mathrm{MnN}_{10} \mathrm{O}_{8} \mathrm{Cu} \\
(853.97)\end{array}$ & Pink & 330 & 38 & $\begin{array}{c}43.60 \\
(43.65)\end{array}$ & $\begin{array}{c}2.83 \\
(2.86)\end{array}$ & $\begin{array}{l}16.40 \\
(16.45)\end{array}$ \\
\hline$[\mathrm{C}$ & $\begin{array}{c}\mathrm{C}_{31} \mathrm{H}_{24} \mathrm{Cl}_{2} \mathrm{CoN}_{10} \mathrm{O}_{8} \mathrm{Ni} \\
(853.14)\end{array}$ & & 338 & 4( & $\begin{array}{c}43.63 \\
(43.68)\end{array}$ & $\begin{array}{c}2.84 \\
(2.88)\end{array}$ & $\begin{array}{c}16.42 \\
(16.44)\end{array}$ \\
\hline$[\mathrm{C}$ & $\begin{array}{r}\mathrm{C}_{31} \mathrm{H}_{24} \mathrm{Cl}_{2} \mathrm{C} \\
(857\end{array}$ & Dirty & 320 & 41 & $\begin{array}{c}43.40 \\
(43.44)\end{array}$ & $\begin{array}{c}2.82 \\
(2.85)\end{array}$ & $\begin{array}{c}16.33 \\
(16.38)\end{array}$ \\
\hline$\left[\mathrm{NiL}_{1} \mathrm{~F}\right.$ & $\begin{array}{c}\mathrm{C}_{31} \mathrm{H}_{24} \mathrm{Cl}_{3} \mathrm{FeN}_{10} \mathrm{O}_{8} \mathrm{Ni} \\
(885.60)\end{array}$ & Brown & 3 & 29 & $\begin{array}{c}42.04 \\
(42.08)\end{array}$ & $\begin{array}{c}2.73 \\
(2.76)\end{array}$ & $\begin{array}{c}15.82 \\
(15.86)\end{array}$ \\
\hline$\left[\mathrm{CuL}_{1} \mathrm{FeL}_{2} \mathrm{Cl}_{2}\right] \mathrm{Cl}$ & $\begin{array}{c}\mathrm{C}_{31} \mathrm{H}_{24} \mathrm{Cl}_{3} \mathrm{FeN}_{10} \mathrm{O}_{8} \mathrm{Cu} \\
(890.40)\end{array}$ & $\begin{array}{l}\text { Reddish } \\
\text { Brown }\end{array}$ & 348 & 35 & $\begin{array}{c}41.81 \\
(41.84)\end{array}$ & $\begin{array}{c}2.72 \\
(2.76)\end{array}$ & $\begin{array}{l}15.73 \\
(15.76)\end{array}$ \\
\hline
\end{tabular}

Figure 1. Heterobinuclear complexes of the type $\left[\mathrm{ML}_{1} \cdot \mathrm{M}^{\prime} \mathrm{L}_{2} \mathrm{Cl}_{2}\right]$

\section{IR spectra of the heterobinuclear complexes}

The relevant IR bands and their assignments are shown in Table 2. The IR spectra of the binuclear complexes under investigation show several bands belonging to ligands $\mathrm{L}_{1}$ and $\mathrm{L}_{2}$. They are considerably changed compared with the relevant bands of the ligands and monometallic complexes $^{16}$. Results given in table are consistent with the some previous results ${ }^{17-22}$.

Table 2. IR Spectral data, $\mathrm{cm}^{-1}$ of the heterobinuclear complexes

\begin{tabular}{ccccccccc}
\hline Complexes & $\begin{array}{c}v \\
(\mathrm{C}=\mathrm{O})\end{array}$ & $\begin{array}{c}v \\
(\mathrm{~N}-\mathrm{H})\end{array}$ & $\begin{array}{c}\text { Ring } \\
\text { Stretching }\end{array}$ & $\begin{array}{c}v\left(\mathrm{NO}_{2}\right) \\
(\text { Asym/Sym })\end{array}$ & $\begin{array}{c}v \\
(\mathrm{C}=\mathrm{N})\end{array}$ & $\begin{array}{c}N \\
(\mathrm{M}-\mathrm{N})\end{array}$ & $\begin{array}{c}N \\
(\mathrm{M}-\mathrm{O})\end{array}$ & $\begin{array}{c}N \\
(\mathrm{M}-\mathrm{Cl})\end{array}$ \\
\hline$\left[\mathrm{MnL}_{1} \mathrm{NiL}_{2} \mathrm{Cl}_{2}\right]$ & 1722 & 3320 & 1616 & $1532 / 1388$ & 1618 & 485 & 478 & 322 \\
{$\left[\mathrm{MnL}_{1} \mathrm{CuL}_{2} \mathrm{Cl}_{2}\right]$} & 1714 & 3324 & 1618 & $1526 / 1382$ & 1614 & 474 & 472 & 318 \\
{$\left[\mathrm{CoL}_{1} \mathrm{NiL}_{2} \mathrm{Cl}_{2}\right]$} & 1742 & 3332 & 1612 & $1532 / 1398$ & 1632 & 472 & 472 & 324 \\
{$\left[\mathrm{CoL}_{1} \mathrm{CuL}_{2} \mathrm{Cl}_{2}\right]$} & 1745 & 3328 & 1624 & $1538 / 1388$ & 1626 & 465 & 468 & 326 \\
{$\left[\mathrm{NiL}_{1} \mathrm{FeL}_{2} \mathrm{Cl}_{2}\right] \mathrm{Cl}$} & 1718 & 3322 & 1638 & $1570 / 1348$ & 1572 & 468 & 472 & 320 \\
{$\left[\mathrm{CuL}_{1} \mathrm{FeL}_{2} \mathrm{Cl}_{2}\right] \mathrm{Cl}$} & 1738 & 3337 & 1630 & $1564 / 1352$ & 1576 & 475 & 464 & 324 \\
\hline
\end{tabular}

\section{Electronic spectra and magnetic moments}

Electronic spectra and magnetic moment value of heterobinuclear complexes are given in Table 3. Magnetic moment values are measured in DMF solvent and show non-electrolyte nature of complexes, except $\left[\mathrm{NiL}_{1} \mathrm{FeL}_{2} \mathrm{Cl}_{2}\right] \mathrm{Cl}$ and $\left[\mathrm{CuL}_{1} \mathrm{FeL}_{2} \mathrm{Cl}_{2}\right] \mathrm{Cl}$ which are 1:1 electrolyte. 
The electronic spectra of metal complexes were recorded in DMF solvent and contain mixed transitions due to two different metal ions. The binuclear complexes possess anti-ferromagnetic properties at room temperature by intra-molecular spin exchange interaction between $\mathrm{M}$ and $\mathbf{M}$ metal ions. Results given in Table 3 are consistent with the heterobinuclear complexes ${ }^{23}$.

Table 3. Electronic spectra, magnetic moment and EPR data of heterobinuclear complexes

\begin{tabular}{|c|c|c|c|c|c|}
\hline \multirow{2}{*}{ Complexes } & \multirow{2}{*}{$\begin{array}{l}\text { Transition, } \mathrm{cm}^{-1} \\
\left(\text { values, } \mathrm{cm}^{-1} \mathrm{M}^{-1} \text { ) }\right.\end{array}$} & \multirow{2}{*}{ Assignments } & \multirow{2}{*}{$\begin{array}{c}\mu_{\text {eff }} \\
\text { (B.M.) }\end{array}$} & \multicolumn{2}{|c|}{ EPR Value } \\
\hline & & & & $\mathrm{g}_{\|}$ & $\mathbf{g}_{\perp}$ \\
\hline$\left[\mathrm{MnL}_{1} \mathrm{NiL}_{2} \mathrm{Cl}_{2}\right]$ & $\begin{array}{c}20,018(304) \\
18,182(264) \\
12,508(46)\end{array}$ & $\begin{array}{c}{ }^{4} \mathrm{~A}_{2} \mathrm{~g} \rightarrow{ }^{4} \mathrm{~T}_{1} \mathrm{~g}(\mathrm{P}) \\
{ }^{4} \mathrm{~A}_{2} \mathrm{~g} \rightarrow{ }^{4} \mathrm{~T}_{1} \mathrm{~g} \\
{ }^{4} \mathrm{~A}_{2} \mathrm{~g} \rightarrow{ }^{4} \mathrm{~T}_{2} \mathrm{~g}\end{array}$ & 3.88 & & \\
\hline$\left[\mathrm{MnL}_{1} \mathrm{CuL}_{2} \mathrm{Cl}_{2}\right]$ & $\begin{array}{c}38,322(58) \\
25,444(426) \\
20,410 \\
16592(410)\end{array}$ & $\begin{array}{c}\text { C.T. } \\
{ }^{4} \mathrm{~A}_{1 \mathrm{~g}}(\mathrm{G})={ }^{6} \mathrm{~A}_{1 \mathrm{~g}} \\
{ }^{4} \mathrm{~T}_{2 \mathrm{~g}}(\mathrm{G})={ }^{6} \mathrm{~A}_{1 \mathrm{~g}} \\
{ }^{2} \mathrm{E}_{\mathrm{g}}(\mathrm{G})={ }^{6} \mathrm{~B}_{1 \mathrm{~g}} \\
{ }^{4} \mathrm{~T}_{1 \mathrm{~g}}(\mathrm{G})={ }^{6} \mathrm{~A}_{1 \mathrm{~g}}\end{array}$ & 5.14 & $\begin{array}{c}1.9 \\
1\end{array}$ & $\begin{array}{c}1.8 \\
6\end{array}$ \\
\hline$\left[\mathrm{CoL}_{1} \mathrm{NiL}_{2} \mathrm{Cl}_{2}\right]$ & $\begin{array}{c}6,568(3.1) \\
14,418(5.4) \\
21,268(3.4) \\
16,376(2.1)\end{array}$ & $\begin{aligned}{ }^{4} \mathrm{~T}_{2 \mathrm{~g}}(\mathrm{~F}) & -{ }^{6} \mathrm{~T}_{1 \mathrm{~g}}(\mathrm{~F}) \\
{ }^{4} \mathrm{~A}_{\mathrm{g}}(\mathrm{F}) & \rightarrow{ }^{4} \mathrm{~T}_{1 \mathrm{~g}}(\mathrm{~F}) \\
{ }^{2} \mathrm{~A}_{1 \mathrm{~g}} & \rightarrow{ }^{1} \mathrm{~B}_{1 \mathrm{~g}} \\
{ }^{1} \mathrm{~A}_{1 \mathrm{~g}} & \rightarrow{ }^{1} \mathrm{~B}_{2 \mathrm{~g}}\end{aligned}$ & 1.92 & & \\
\hline$\left[\mathrm{CoL}_{1} \mathrm{CuL}_{2} \mathrm{Cl}_{2}\right]$ & $\begin{array}{c}6,562(3.3) \\
14,416(5.2) \\
18,234(5.3) \\
15,510(6.3) \\
20,304(2.8)\end{array}$ & 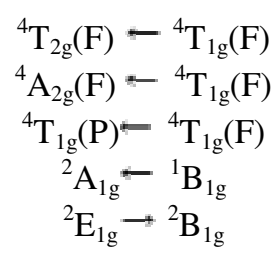 & 1.91 & $\begin{array}{c}1.8 \\
4\end{array}$ & \\
\hline$\left[\mathrm{NiL}_{1} \mathrm{FeL}_{2} \mathrm{Cl}_{2}\right] \mathrm{Cl}$ & $\begin{array}{c}40,466 \\
29,116(28,414) \\
19,540(26,455) \\
15,512(24,270) \\
21,266(3.4) \\
18,784(2.4)\end{array}$ & $\begin{array}{c}\text { C.T. } \\
{ }^{4} \mathrm{E}_{1 \mathrm{~g}}(\mathrm{G}) \leftarrow{ }^{6} \mathrm{~A}_{1 \mathrm{~g}} \\
{ }^{4} \mathrm{~T}_{2 \mathrm{~g}}(\mathrm{G}) \leftarrow{ }^{6} \mathrm{~A}_{1 \mathrm{~g}} \\
{ }^{2} \mathrm{~T}_{1}(\mathrm{G})-{ }^{6} \mathrm{~A}_{1 \mathrm{~g}} \\
{ }^{1} \mathrm{~A}_{1 \mathrm{~g}} \rightarrow{ }^{1} \mathrm{~B}_{1 \mathrm{~g}} \\
{ }^{1} \mathrm{~A}_{1 \mathrm{~g}} \rightarrow{ }^{1} \mathrm{~A}_{2 \mathrm{~g}}\end{array}$ & 5.86 & & \\
\hline$\left[\mathrm{CuL}_{1} \mathrm{FeL}_{2} \mathrm{Cl}_{2}\right] \mathrm{Cl}$ & $\begin{array}{c}40,466 \\
29,113(28,411) \\
15,510(24,241) \\
19,544(26,460) \\
15,508(6.04) \\
18,308(8.4)\end{array}$ & $\begin{array}{c}\text { C.T. } \\
{ }^{4} \mathrm{~A}_{1 \mathrm{~g}}(\mathrm{G}) \leftarrow{ }^{6} \mathrm{~A}_{1 \mathrm{~g}} \\
{ }^{4} \mathrm{~T}_{\mathrm{gg}}(\mathrm{G})-{ }^{6} \mathrm{~A}_{1 \mathrm{~g}} \\
{ }^{4} \mathrm{~T}_{2}(\mathrm{G})={ }^{6} \mathrm{~A}_{1 \mathrm{~g}} \\
{ }^{1} \mathrm{~B}_{1 \mathrm{~g}}(\mathrm{G}) \rightarrow{ }^{2} \mathrm{~A}_{1 \mathrm{~g}} \\
{ }^{2} \mathrm{~B}_{1 \mathrm{~g}}-{ }^{2} \mathrm{E}_{\mathrm{g}}\end{array}$ & 5.88 & & \\
\hline
\end{tabular}




\section{EPR spectra}

EPR spectra value of all the metal complexes was given in Table 3. The EPR spectra of heterobinuclear complexes were recorded at room temperature. The spectra of $\left[\mathrm{MnL}_{1} \mathrm{NiL}_{2} \mathrm{Cl}_{2}\right]$ show $\mathrm{g}_{\|}=1.91, \mathrm{~g}_{\perp}=1.86$ which show square planer $\mathrm{Mn}$ (II) complexes. The signals for the two different metals are merged together and new signals are obtained.

\section{Antimicrobial activity}

In vitro antimicrobial activity of heterobinuclear metal complexes have been tested against the bacteria Bacillus subtilis and Escherichia coli and fungi Aspirgillus niger and Aspirgillus flavus and are summarized in Table 4. The values indicate that all complexes have higher antimicrobial activity than the free ligand. Such increased activity of the metal chelates can be explained on the basis of chelation theory. On chelation, the polarity of the metal ion will be reduced to a greater extent due to overlap of the ligand orbital and partial sharing of the positive charge of the metal ion with donor groups. Further, it increases the delocalization of $\pi$-electrons over the whole chelate ring and enhances the penetration of the complexes into lipid membranes and blocking of the metal binding sites in enzymes of microorganism. These complexes also disturb the respiration process of the cell and thus block the synthesis of proteins, which restricts further growth of microorganism ${ }^{24}$.

Table 4. Antibacterial and antifungal activity of heterobinuclear metal complexes

\begin{tabular}{|c|c|c|c|c|c|}
\hline \multirow{2}{*}{ Compounds } & \multirow{2}{*}{$\begin{array}{l}\text { *Conc. } \\
\mu \mathrm{g} \mathrm{moL}^{-1}\end{array}$} & \multicolumn{2}{|c|}{ Bacterial Inhibition, $\%$} & \multicolumn{2}{|c|}{ Antifungal Inhibition, $\%$} \\
\hline & & B. subtilis & E. coli & A. niger & A.flavus \\
\hline \multirow{2}{*}[\mathrm{MnL}_{1}\cdot\mathrm{NiL}_{2}\mathrm{Cl}_{2}]{} & 100 & 42 & 55 & 66 & 59 \\
\hline & 500 & 46 & 60 & 72 & 72 \\
\hline \multirow{2}{*}[\mathrm{MnL}_{1}\cdot\mathrm{CuL}_{2}\mathrm{Cl}_{2}]{} & 100 & 44 & 48 & 74 & 68 \\
\hline & 500 & 55 & 52 & 86 & 81 \\
\hline \multirow{2}{*}[\mathrm{CoL}_{1}\cdot\mathrm{NiL}_{2}\mathrm{Cl}_{2}]{} & 100 & 50 & 70 & 73 & 76 \\
\hline & 500 & 58 & 78 & 82 & 85 \\
\hline \multirow{2}{*}[\mathrm{CoL}_{1}\cdot\mathrm{CuL}_{2}\mathrm{Cl}_{2}]{} & 100 & 44 & 58 & 65 & 72 \\
\hline & 500 & 62 & 70 & 74 & 81 \\
\hline \multirow[t]{2}{*}[\mathrm{NiL}_{1}\cdot\mathrm{FeL}_{2}\mathrm{Cl}_{2}]{$\cdot \mathrm{Cl}$} & 100 & 44 & 60 & 71 & 73 \\
\hline & 500 & 50 & 62 & 78 & 76 \\
\hline \multirow{2}{*}[\mathrm{CuL}_{1}\cdot\mathrm{FeL}_{2}\mathrm{Cl}_{2}]{$\cdot \mathrm{Cl}$} & 100 & 47 & 58 & 61 & 76 \\
\hline & 500 & 56 & 64 & 83 & 82 \\
\hline
\end{tabular}

\section{Acknowledgment}

The authors are thankful to ACBR, Delhi for providing spectral data and SAIF, CDRI, Lucknow for providing elemental analysis data. Authors are also thankful to SARC, Meerut for providing antimicrobial activity.

\section{References}

1. Fenton D E, Biocoordination Chemistry, first Ed., Oxford University Press Oxford, 1995.

2. Karlin K D and Zubieta J, Biological and Inorganic Copper chemistry, Academic Press, New York, 1996. 
3. Khan O, Molecular magnetism, Wiley-VCH, New York, 1993.

4. Gatteschi D, et al., Magnetic Molecular Materials, Kluwev Academic Dordrecht, 1991.

5. Karlin K D and Tyeklav Z, Bioinorganic Chemistry of Copper, Chapmanand Hall, London, 1993.

6. Tabassum S, Sharma G C, Arjmand F and Azam A, Nanotechnology, 2010, 21,195102.

7. Kobayashi K, Tamura M, Hayashi K, Hori H and Morimoto H, J Biol Chem., 1980, 255, 2239-2242.

8. Kadish K M, Boulas P L, Kisters M, Vogeland E Aukauloo A. M, D'Souza F and Guilard R, Inorg Chem., 1998, 37, 2693-2700.

9. Bag N, Chern S S and Peng S M, Inorg Chem., 1995, 34, 753-756.

10. Kalam A Tripathi V, Srivastava S, Pandey Y, Kumar A, Gupta A, Srivastava S and Purohit A, Turk J Chem., 2010, 34,147-155.

11. Lashanizadegan M and Seraj S, Turk J Chem., 2010, 34, 263-268.

12. Arjmand F, Muddasir M and Khan R H, Eur J Med Chem., 2010, 45, 3549-3557.

13. Novotná R, Zdenek Travnicek and Popa I, Inorg Chem Acta., 2010, 363, 2071-2079.

14. Bandini A L, Banditelli G, Minghetti G and Bonati F, Canadian J Chem., 1979, 57, 3237-3242.

15. Rajavel R, Senthil Vadivu M and Anitha C, E-J Chem., 2008, 5(3), 620-626.

16. Woo L K and Maurya M R, Inorg Chem., 1991, 30, 4671-4672.

17. Shah N and Kar S K, J Inorg Nucl Chem., 1997, 39, 1236-1238.

18. Ferraro J R, Plenum Press, New York, 1971.

19. Campell M J M, Grzeskowiak R and Juneja S G, J Inorg Nucl Chem., 1974, 36, 2485-2488.

20. Folgado J V et al., J Chem Sci Dalton Trans., 1986, 1061-1064.

21. Shyama A S and Yang F, Am J Sci Res, 2009, 5, 20-26.

22. Sonmez M, Erciyes University Fen Bilimleri Enstitusu Dergisi, 2008, 24(1-2), 308-314.

23. Sakamoto M, Motoko K, Hiroshi S, Yuzo N, Yoshihiko S, Akira M, Masaaki O and Hisashi O, Synthesis Reactivity of Inorganic Metal Organic Chemistry, 1997, 27(4), 567-575.

24. Dharmaraj N, Viswanathamurthi P and Natarajan K, Trans Met Chem., 2002, 26, 105. 


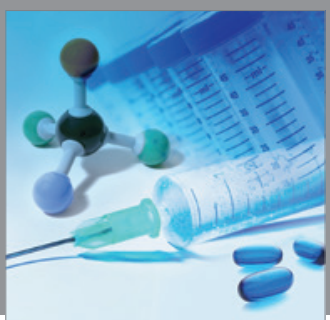

International Journal of

Medicinal Chemistry

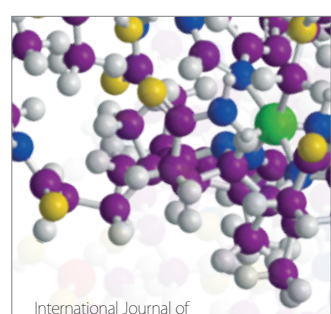

Carbohydrate Chemistry

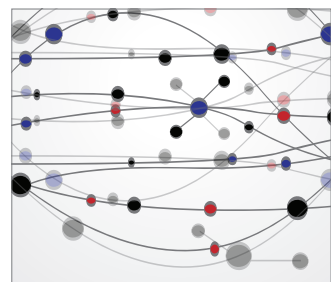

The Scientific World Journal
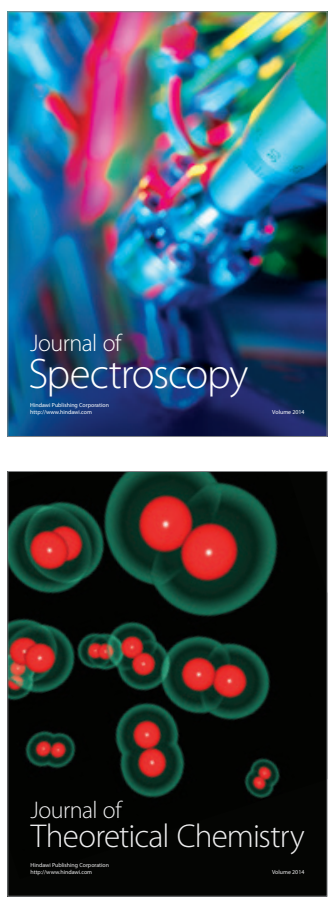
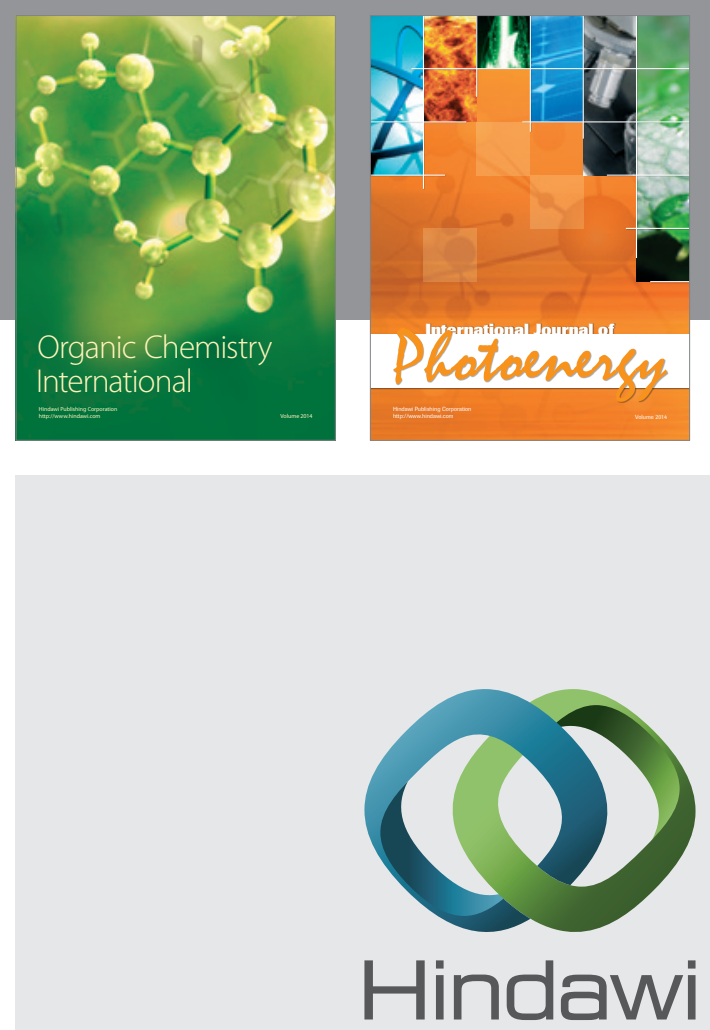

Submit your manuscripts at

http://www.hindawi.com
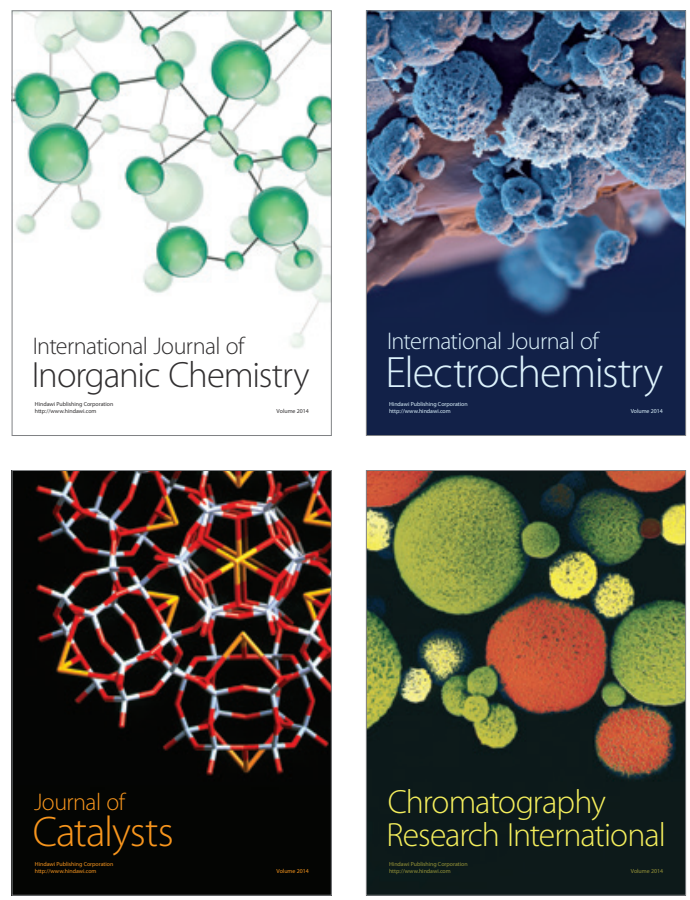
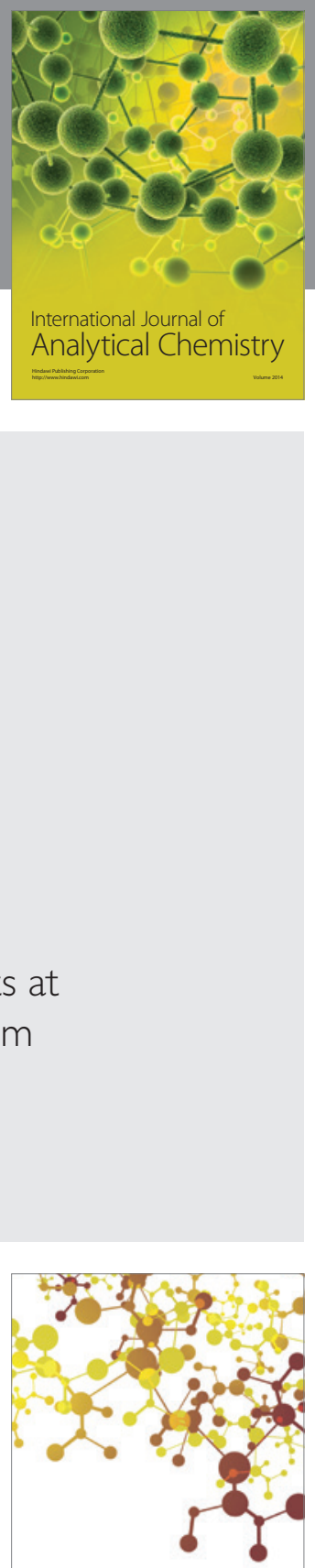

Journal of

Applied Chemistry
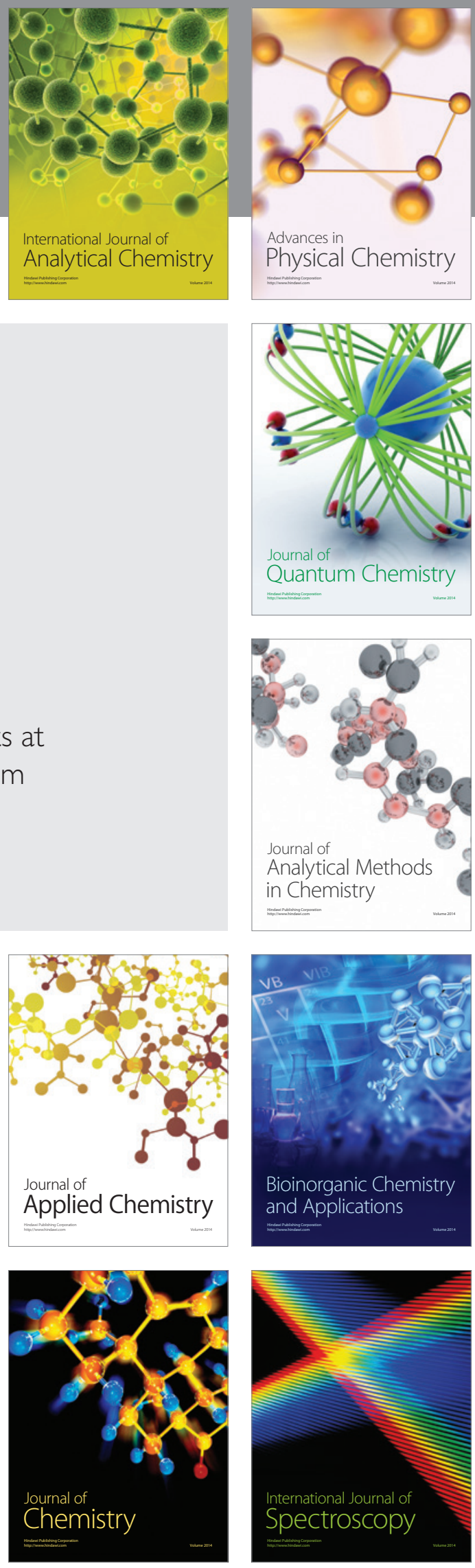